Provided for non-commercial research and education use. Not for reproduction, distribution or commercial use.

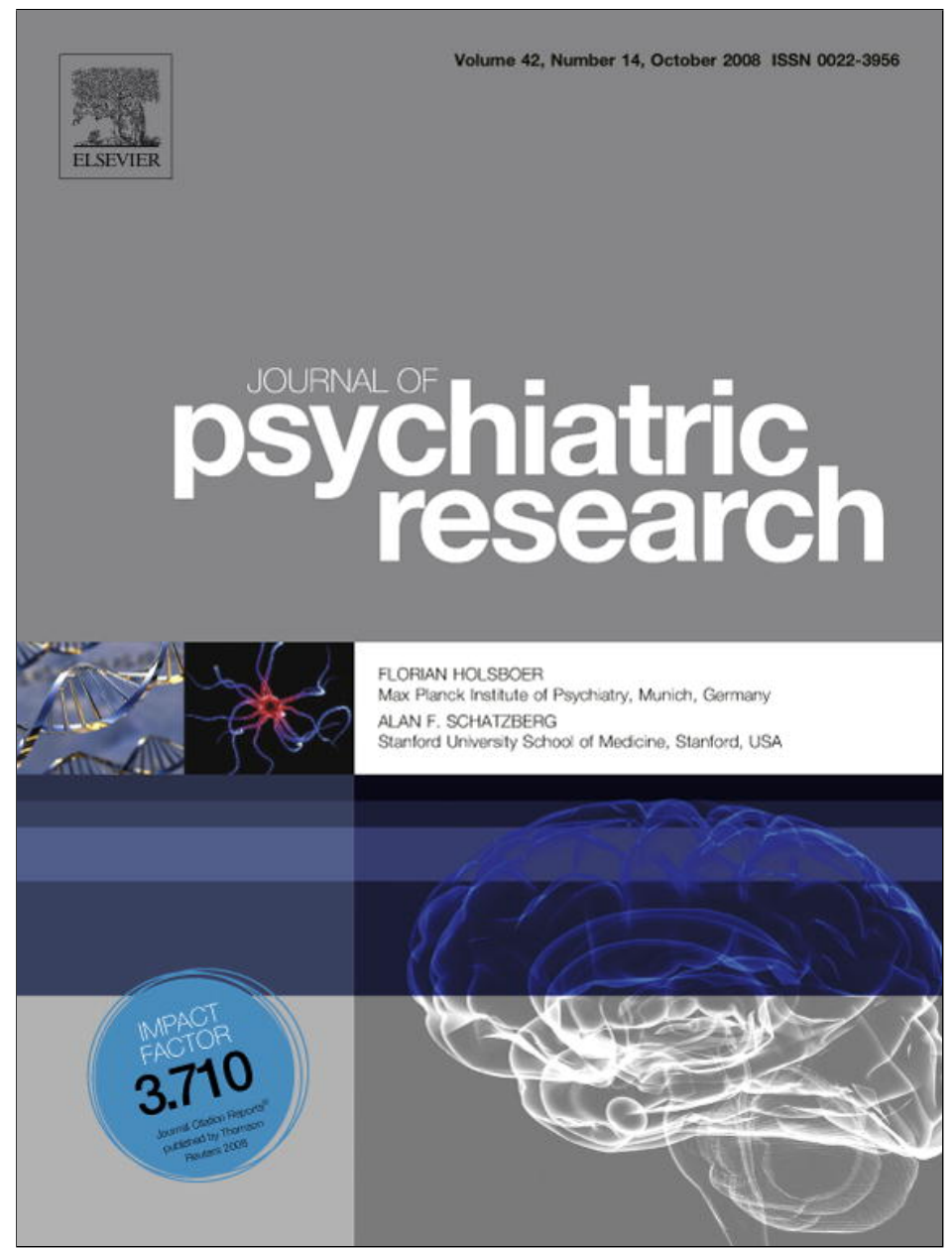

This article appeared in a journal published by Elsevier. The attached copy is furnished to the author for internal non-commercial research and education use, including for instruction at the authors institution and sharing with colleagues.

Other uses, including reproduction and distribution, or selling or licensing copies, or posting to personal, institutional or third party websites are prohibited.

In most cases authors are permitted to post their version of the article (e.g. in Word or Tex form) to their personal website or institutional repository. Authors requiring further information regarding Elsevier's archiving and manuscript policies are encouraged to visit:

http://www.elsevier.com/copyright 


\title{
What explains the relation between family poverty and childhood depressive symptoms?
}

\author{
Melissa Tracy ${ }^{\mathrm{a}}$, Frederick J. Zimmerman ${ }^{\mathrm{b}}$, Sandro Galea ${ }^{\mathrm{a}, *}$, \\ Elizabeth McCauley ${ }^{\mathrm{c}}$, Ann Vander Stoep ${ }^{\mathrm{c}, \mathrm{d}}$ \\ a Department of Epidemiology, University of Michigan, School of Public Health, 109 Observatory Street, Ann Arbor, MI 48109, United States \\ ${ }^{\mathrm{b}}$ Department of Health Services, University of Washington, School of Public Health and Community Medicine, Seattle, WA, United States \\ ${ }^{\mathrm{c}}$ Department of Psychiatry, Division of Child and Adolescent Psychiatry, University of Washington, School of Medicine, Seattle, WA, United States \\ ${ }^{\mathrm{d}}$ Department of Epidemiology, University of Washington, School of Public Health and Community Medicine, Seattle, WA, United States
}

Received 9 May 2007; received in revised form 11 December 2007; accepted 17 January 2008

\begin{abstract}
The relation between low socioeconomic status (SES) and depression has been well documented in adult populations. A number of studies suggest that family SES may be associated with depression among children and adolescents as well, although the evidence is mixed. We assessed the relation between family income and depressive symptoms among 457 children aged 11-13 years and examined pathways that may explain this relation. In-person interviews of children and their caregivers were conducted, including assessment of family income and administration of the Computer-based Diagnostic Interview Schedule for Children (C-DISC). Family income was significantly associated with depressive symptoms, with children in the lowest income group $(<\$ 35,000)$ reporting a mean of 8.12 symptoms compared to 6.27 symptoms in the middle income group $(\$ 35,000-\$ 74,999)$ and 5.13 symptoms in the highest income group $(\geqslant \$ 75,000 ; p<0.001)$. Controlling for the number of stressful life events experienced in the past 6 months attenuated the effect of low family income on depressive symptoms by $28 \%$. Indicators of the family environment explained $45 \%$ and neighborhood median household income and aggravated assault rate explained $12 \%$ of the relation. The family environment, including parental divorce or separation and perceived parental support, appears to explain most of the relation between low family income and childhood depressive symptoms. Further exploration of the pathways between family SES and depression may suggest potential interventions to reduce the occurrence and persistence of depressive symptoms in children.
\end{abstract}

(c) 2008 Elsevier Ltd. All rights reserved.

Keywords: Depressive symptoms; Socioeconomic status; Stressful events; Divorce; Neighborhood; Children

\section{Introduction}

Depression is a common health concern among children and adolescents, with prevalence estimates ranging from $0.4 \%$ to $2 \%$ in children aged $6-12$ years and $2-8.3 \%$ in adolescents aged 13-18 years (Birmaher et al., 1996; Kubik et al., 2003; Ryan, 2005). About $10-15 \%$ of children report a moderate to severe level of depressive symptoms at any

\footnotetext{
* Corresponding author. Tel.: +1 734647 9741; fax: +1 7347635706

E-mail address: sgalea@umich.edu (S. Galea).
}

given time (Nolen-Hoeksema et al., 1992), and between $14 \%$ and $25 \%$ of children and adolescents will experience at least one episode of major depression before adulthood (Kessler and Walters, 1998; Lewinsohn et al., 1998). Depression in childhood or adolescence is associated with a high likelihood of recurrence later in life (Belsher and Costello, 1988; Pine et al., 1998) and is associated with an increased likelihood of adverse outcomes including substance use problems, poor academic performance including failure to complete secondary school, employment difficulties, and teenage childbearing (Dooley et al., 1994; Kelder et al., 2001; Kubik et al., 2003; Schraedley et al., 1999; 
Vander Stoep et al., 2002). Depressed children and adolescents are also at increased risk for suicidal ideation and other comorbid psychiatric disorders (Brady and Kendall, 1992; Kubik et al., 2003).

The relation between low socioeconomic status (SES) and depression has been well documented in adult populations (Lorant et al., 2003). A number of studies suggest that family SES is also associated with depression among children and adolescents (Call and Nonnemaker, 1999; Goodman et al., 2003; Goodman, 1999; Kubik et al., 2003; McLeod and Shanahan, 1993; Roberts et al., 1997; Schraedley et al., 1999; Wade, 2001). However, one large meta-analysis found no correlation between SES and scores on the Children's Depression Inventory among 816 year olds (Twenge and Nolen-Hoeksema, 2002), and one study found no relation between maternal SES and high levels of depressive symptoms in 10-year-olds when other risk factors for depression were considered (Leech et al., 2006). Additionally, some studies have found the relation between SES and childhood depression to be confined to particular subgroups within the child population, like those with insecure attachment to their parents (Graham and Easterbrooks, 2000).

Three plausible pathways may explain a relation between lower family SES and childhood depression. First, children in lower SES families may be exposed to more traumatic events and stressors (e.g., witnessing violence, frequent moves) (Costello et al., 2003; McLoyd, 1998), which in turn may increase their risk of depressive symptoms (Franko et al., 2004; Nolen-Hoeksema et al., 1992; Patton et al., 2003; Schraedley et al., 1999; Silberg et al., 1999). Thus, using the framework developed Kraemer et al. (2001) to describe how two risk factors work together to influence an outcome, we hypothesize that stressful life events may at least partially mediate the relation between low family income and depression (Fig. 1a).

Second, the strain of financial stress may lead to family conflict and potentially disruption (e.g., divorce or separation of parents) (Gilman et al., 2003; Takeuchi et al., 1991; Wade, 2001), or may influence parenting behaviors, including increased use of harsh discipline methods, lack of affection and support, or inadequate supervision (Bolger et al., 1995; Bradley and Corwyn, 2002; Costello et al., 2003; McLeod and Shanahan, 1993; McLoyd, 1998) which can, in turn, affect the child's mental health status (Black and Pedro-Carroll, 1993; Kim et al., 2003; McLeod and Shanahan, 1993). Conversely, parental divorce or separation may lead to decreased family income, as single-parent families typically have lower incomes (Aseltine, 1996; McQuillan, 1992). Thus, we hypothesize that the family environment, including parental divorce or separation, household composition, and parental support, and family SES are overlapping risk factors (Kraemer et al., 2001) for depressive symptoms in children and adolescents (Fig. 1b).

Third, children in families with low SES may be more likely than those in higher SES families to reside in more disadvantaged neighborhoods with higher levels of crime,
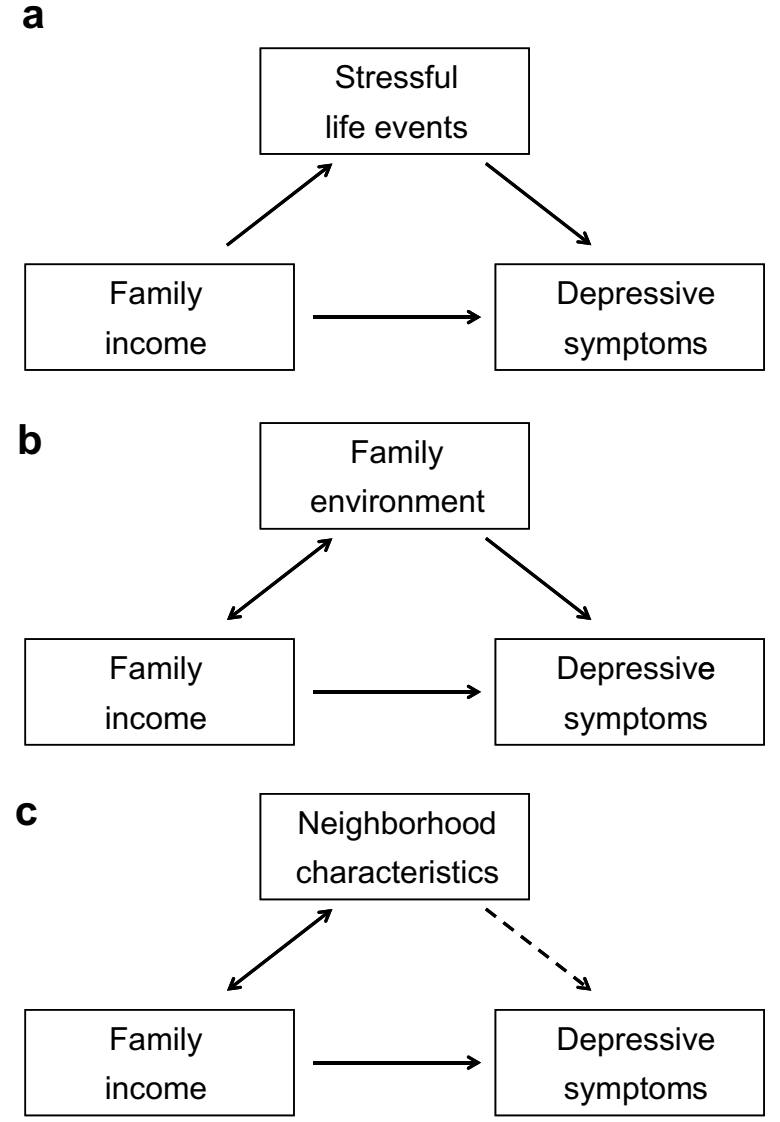

Fig. 1. Theorized relations between family income, stressful life events, family environment, neighborhood characteristics, and depressive symptoms in children and adolescents. (a) Stressful life events as a mediator of the relation between family income and depressive symptoms. (b) Family environment and family income as overlapping risk factors for depressive symptoms. (c) Neighborhood characteristics as a proxy risk factor for family income, which is related to depressive symptoms.

exposing them to suboptimal physical and social environmental conditions that may adversely influence their mental health (Goodman et al., 2003; Kalff et al., 2001; McLeod and Shanahan, 1993; McLoyd, 1998; Takeuchi et al., 1991). However, little evidence exists as to whether neighborhood characteristics like poverty and violent crime influence depressive symptoms in children and adolescents independent of their association with family SES, as many studies of the relations between neighborhood characteristics and depressive symptoms have not included adjustment for family SES (e.g. (Ozer and Weinstein, 2004)) and few studies have looked explicitly at depressive symptoms, focusing instead on problem behaviors or developmental and academic outcomes (Brooks-Gunn et al., 1993; Kalff et al., 2001; Leventhal and Brooks-Gunn, 2000). We hypothesize that neighborhood characteristics are proxy risk factors (Kraemer et al., 2001) for family income, associated with depressive symptoms only through their relation with family income (Fig. 1c).

We assessed the relation between family income and depressive symptoms among an urban population of 
children aged 11-13 years and examined the potential pathways described above that may explain this relation.

\section{Methods}

\subsection{Participants}

Study participants were sixth grade students enrolled in the Developmental Pathways Project, a longitudinal epidemiologic study assessing depression and conduct problems. Universal mental health screening was carried out with sixth grade students at four Seattle-area public schools, chosen as representative of the Seattle public middle school population (Seattle Public Schools, 2004), in 4 consecutive years; details of the screening procedure have been described elsewhere (Vander Stoep et al., 2005). Each year following screening, a random sample of children, stratified by their scores on the depression and conduct problem screening instruments, was identified for participation in the longitudinal study. Children eligible for the study included those capable of participating in lengthy in-person interviews with a parent or guardian who could also participate in interviews. Of 941 families recruited for the longitudinal study during the 4 years of recruitment, 811 $(86.2 \%)$ were eligible for participation. Ineligibility of families recruited for participation in the longitudinal study stemmed from different eligibility requirements for participation in the mental health screening; specifically, students with mild disabilities and those without a parent or other caregiver who had adequate English proficiency were eligible for participation in mental health screening but not for participation in the longitudinal study. Additionally, students who moved out of Seattle and the immediately surrounding areas between the time of mental health screening and recruitment were not eligible for participation in the longitudinal study since multiple in-person interviews were required over several years. Five hundred twenty-one eligible families $(64.2 \%)$ agreed to participate in the longitudinal study. Non-participants were less likely to be white and in the gifted educational program at Seattle public middle schools than participants, and were more likely to have a parent born outside of the United States or Canada. However, there were no differences between those who did and did not participate with regard to gender, Hispanic ethnicity, number of adults reported to be living in the household, and, importantly, mean depression symptoms as measured by the Mood and Feelings Questionnaire (Angold and Costello, 1987) or conduct problems as measured by the externalizing items of the Youth Self Report (Achenbach, 2001) at the time of screening. The Institutional Review Board of the University of Washington reviewed and approved the study.

In-person interviews of participating children and their primary caregiver were conducted by trained interviewers, with baseline interviews taking place an average of three months after the initial mental health screening, and additional interviews conducted every 6 months thereafter, for a total of five interviews. Data for this analysis come from the baseline interview of the longitudinal study, conducted between April 22, 2002 and May 4, 2005. This analysis was restricted to students who lived in Seattle at the time of the baseline interview and who could be geocoded to their neighborhood of residence.

\subsection{Measures}

\subsubsection{Dependent variable}

Depressive symptoms were evaluated at the baseline interview via the depression module of the Computerbased Diagnostic Interview Schedule for Children (CDISC) (Costello et al., 1985; NIMH, 1997; Schwab-Stone et al., 1996; Shaffer et al., 2000), which is based on the Diagnostic and Statistical Manual of Mental Disorders, 4th edition (American Psychiatric Association, 1994). This diagnostic interview has acceptable internal consistency, test-retest reliability, and criterion validity (Schwab-Stone et al., 1996; Shaffer et al., 2000), and the computerized version of the depression module has been shown to have high agreement with physician assessments of depression (Cawthorpe, 2001). The C-DISC has been used in a number of studies assessing depression in children and adolescents (King and Sobolewski, 2006; Shaffer et al., 2004; Slesnick and Prestopnik, 2005).

Since the prevalence of major depressive disorder was low in this sample, total symptom count from the depression module of the C-DISC, as reported by the child, was used as the main outcome of interest. All of the symptoms considered positive in the depressive symptom count were reported to be present "most of the day", "almost every day" in a twoweek period during the past year (Shaffer et al., 2000). Thus, duration and simultaneity criteria were included in generating the symptom count. Each of the nine criteria for major depression was represented by varying numbers of items (depressed mood: 2 items; diminished interest or pleasure in activities: 1 item; weight loss/gain or increased/decreased appetite: 4 items; insomnia or hypersomnia: 2 items; psychomotor agitation or retardation: 2 items; fatigue or loss of energy: 2 items; feelings of worthlessness or excessive guilt: 2 items; diminished ability to concentrate or increased indecisiveness: 3 items; and recurrent thoughts about death or suicidal ideation: 3 items), for a possible total symptom count of 22 (NIMH, 2003). The use of symptom count rather than presence of disorder as the outcome of interest has been demonstrated in several other studies using other diagnostic instruments (Costello et al., 2003; Silberg et al., 1999). While one of these studies used symptom counts combining reports from both children and parents (Costello et al., 2003), the other used child-reported symptoms alone (Silberg et al., 1999). Numerous studies have suggested that internalizing symptoms like those manifested in depression are better reported by the child or adolescent him- or herself in community samples (Angold et al., 1987), especially at older ages like those included in this analysis (Edelbrock et al., 1985), whereas externalizing symptoms may be better reflected in 
the reports of caregivers (Edelbrock et al., 1986); therefore we chose to use child-reported symptom count in this analysis.

\subsubsection{Independent variable}

Family income was reported by the primary caregiver at the baseline visit and was used as a measure of household SES in this analysis. Family income was regrouped from the original ten categories presented to the primary caregiver into three categories for this analysis $(<\$ 35,000$, $\$ 35,000-\$ 74,999$, and $\geqslant \$ 75,000$ ), corresponding to tertiles of family income within the sample.

\subsubsection{Adjustment variables}

Demographic information for the child, including age, gender, and race/ethnicity, was obtained from school records at the time of mental health screening. Because of high levels of comorbidity between depressive and anxiety disorders (Brady and Kendall, 1992), we also considered adjustment for anxiety symptoms, in order to evaluate the relation between family income and depressive symptoms over and above any effect of anxiety on depressive symptoms. We used the total score from the child-report Multidimensional Anxiety Scale for Children (MASC) (March et al., 1997), which consists of 39 items scored on a Likert scale (Ivarsson, 2006). The MASC has shown high levels of internal consistency, test-retest reliability, and some ability to distinguish between anxiety and depressive disorders (Baldwin and Dadds, 2007; Dierker et al., 2001; Ivarsson, 2006; March et al., 1997; March et al., 1999; Rynn et al., 2006). The internal consistency of the MASC total score in our sample was 0.87 .

\subsubsection{Pathway 1 - Stressful life events}

Information on life events in the past six months was collected using 31 items from the Life Events Checklist (Johnson and McCutcheon, 1980), which has been found to be reliable and valid for child populations in the United States (Johnson and Sarason, 1979). For each item (e.g., "a new baby brother or sister was born into your family", "you moved to a new home", "somebody close to you died"), children were asked to report whether they had experienced the event in the past 6 months. The number of events reported to have occurred in the past 6 months was summed for each participant, and this sum was categorized into thirds $(0-3,4-5$, and $\geqslant 6$ stressful life events). Three events related to family disruption ("your parents got divorced or separated", "your parents/guardians had more arguments", and "you argued a lot more with your parents/guardians or family members") were excluded from this sum, so as to keep this pathway distinct from Pathway 2.

\subsubsection{Pathway 2 - Family environment}

Marital status of the participant's parents was assessed during the baseline interview. The number of adults living in the household was also reported by the primary care- giver at the baseline interview and was categorized as one versus two or more adults; it has been hypothesized that psychological well-being may differ among adolescents residing in single-parent households and those residing in households comprised of one parent and other adults (Zimmerman et al., 1995). The parental support subscale score from the Social Support Scale for Children (Harter, 1985), as reported by the child, was used as a measure of parental support $(\alpha=0.80$ in this sample). This subscale consists of six questions assessing children's perceptions of the degree to which their parents understand them, want to hear about their problems, care about their feelings, treat them like a person who really matters, like them the way they are, and act like what they do is important. Higher scores indicate greater levels of parental support. Since perceptions of parental support may be influenced by levels of depressive symptoms, we also considered a more objective measure of family support, created by averaging participants' responses to three questions assessing the frequency with which their parents or other adults in their families spent time with them doing something fun, talked with them or listened to their ideas, and ate meals with them in the four weeks prior to the interview (with response choices of 0 days, 1-3 days, 4-6 days, 7-14 days, and $15-28$ days).

\subsubsection{Pathway 3 - Neighborhood characteristics}

All participants' residential addresses were geocoded to their Community Reporting Area of residence (Department of Neighborhoods, City of Seattle, USA). These 53 neighborhood units, comprised of aggregations of census tracts, were developed by the Department of Neighborhoods of the City of Seattle and have been used to track trends in health behaviors and demographics over time in Seattle neighborhoods and to identify areas in need of intervention (Department of City Planning, City of Seattle, 2000; Department of Neighborhoods, City of Seattle, 2000). Census 2000 data on neighborhood median household income for each Community Reporting Area were collected from the Department of City Planning of the City of Seattle (2000). Data on the number of crimes committed in each census tract in 2000 were obtained from the Seattle Police Department (2000) and aggregated to the neighborhood level, and crime rates were calculated per 1000 population using the 2000 Census population counts for each neighborhood (Department of City Planning, City of Seattle, 2000). The aggravated assault rate was used as an indicator of violent crime. Aggravated assault is defined as "an unlawful attack by one person upon another for the purpose of inflicting severe or aggravated bodily injury... usually accompanied by the use of a weapon or by means likely to produce death or great bodily harm" (FBI, 2004).

\subsection{Statistical analysis}

Weights were developed and applied to correct for oversampling of children who screened high for depression and 
conduct problems and to make the sample demographically similar to the Seattle public middle schools population with respect to gender, race/ethnicity, and educational program distribution (e.g., regular, gifted, special education) (Seattle Public Schools, 2004). All participants were geocoded to their census tract and their Community Reporting Area of residence. We calculated the mean number of depressive symptoms in each category of the covariates of interest, using two-tailed $t$-tests and analysis of variance to test for associations between covariates, family income, and depressive symptoms. We also fit unadjusted linear regression models, predicting depressive symptoms from each covariate separately. We used twotailed $\chi^{2}$ tests to compare the distribution of each covariate of interest within the three levels of family income, and fit unadjusted polytomous logistic regression models to assess the relation between covariates and family income, with high levels of family income $(\geqslant \$ 75,000)$ serving as the referent outcome. Each of the three hypothesized pathways was tested separately, building on a base model including family income and adjusted for the participant's gender, race/ethnicity, and age. A final multivariable model was fit including covariates from all three pathways. generalized estimating equations (GEE) were used to adjust for correlation between individuals in the same neighborhoods and schools. The percent change in the regression coefficient for low family income was calculated for each model, in order to gauge the effect of each pathway on the relation between family income and depressive symptoms. Likelihood ratio tests were conducted to determine if each pathway contributed to the overall fit of the model compared to the base model, guiding the interpretation of any change in the regression coefficient for low family income between models. Because the dependent variable was a count of depressive symptoms, analyses were replicated using Poisson regression. Since results were essentially equivalent, the linear regression GEE models are presented here for ease of interpretation. All analyses were carried out using SAS and SUDAAN (Shah et al., 1997).

Sensitivity analyses were conducted to assess the robustness of the results. Multivariable models were fit including anxiety symptoms in the base model in addition to demographic characteristics. Additionally, models were re-run replacing the parental support subscale in the family environment pathway (Pathway 2) with a more objective measure of family involvement.

\section{Results}

\subsection{Characteristics of the study sample}

Overall, of the 521 participants in the longitudinal study, 457 lived in Seattle at the time of the baseline interview and could be geocoded to their neighborhood of residence; the remaining 64 participants were excluded from further analyses. The included participants were representative of the general Seattle public middle schools popula- tion (Seattle Public Schools, 2004), being 51.0\% male, 42.7\% white, $22.5 \%$ African American, 10.5\% Hispanic, $24.3 \%$ Asian or other race, and ranging in age from 11 to 13 years (Table 1$)$.

\subsection{Relations between covariates of interest and depressive symptoms}

The mean depressive symptom count in our sample was 6.54 , with the mean plus one standard deviation estimated to be 11.18 (Table 1). Family income was significantly associated with depressive symptoms, with higher mean levels of symptoms reported by children in lower income families (Table 1; Fig. 2). All of the variables in the three pathways were significantly associated with depressive symptoms: higher numbers of life events in the past 6 months, having divorced or separated (versus married) parents, lower levels of parental support, low neighborhood median income, and high neighborhood aggravated assault rate were associated with higher levels of depressive symptoms, whereas having two or more adults (versus one adult) living in the household was associated with lower levels of depressive symptoms (Table 1). Race/ethnicity was also significantly associated with depressive symptoms, such that African Americans, Hispanics, and those of Asian or other race had higher mean symptom counts than Whites.

\subsection{Relations between covariates of interest and family income}

All of the variables in the three hypothesized pathways were also significantly associated with family income: children from low income families reported experiencing a greater number of life events and were more likely to have divorced or separated parents, to live with only one adult, to report low levels of parental support, and to live in neighborhoods with lower median income and higher aggravated assault rates than children from high income families (Table 2).

\subsection{Multivariable models predicting depressive symptoms}

In the base multivariable model adjusted for demographic characteristics (gender, race/ethnicity, and age), low family income was a significant predictor of higher levels of depressive symptoms (Base Model; $b=2.33$ for low vs. high family income; $p=0.001$ ) (Table 3 ). The effect of low family income was attenuated by $28 \%$ after adjusting for number of life events in the past 6 months (Pathway 1; $b=1.68$ for low vs. high family income; $p=0.023$ ). Adjusting the base model for measures of the family environment changed the effect of low family income on childhood depressive symptoms by $45 \%$, and the effect of low family income was no longer statistically significant (Pathway 2: $b=1.27$ for low vs. high family income; $p=0.151$ ). The relation between low family income and depressive symptoms was also attenuated after adjusting for neighborhood 
Table 1

Distribution of covariates of interest in the study sample, mean depressive symptoms in categories of covariates, and bivariate relations between covariates and depressive symptoms

\begin{tabular}{|c|c|c|c|c|c|c|}
\hline & \multicolumn{2}{|c|}{ Total } & \multicolumn{4}{|c|}{ C-DISC symptom count } \\
\hline & $N$ & $\%$ & Mean $(\mathrm{SE})^{\mathrm{a}}$ & $b(\mathrm{SE})^{\mathrm{b}}$ & $p$-value & Wald $p$-value ${ }^{\mathrm{c}}$ \\
\hline Total & 457 & & $6.54(0.23)$ & & & \\
\hline \multicolumn{7}{|l|}{ Family income } \\
\hline High $(\geqslant \$ 75,000)$ & 146 & 33.5 & $5.13(0.37)$ & 0.00 & - & $<0.001$ \\
\hline Medium $(\$ 35,000-\$ 74,999)$ & 158 & 34.5 & $6.27(0.39)$ & $1.14(0.53)$ & 0.033 & \\
\hline Low $(<\$ 35,000)$ & 143 & 32.0 & $8.12(0.44)$ & $2.98(0.55)$ & $<0.001$ & \\
\hline \multicolumn{7}{|c|}{ Demographic characteristics of students } \\
\hline \multicolumn{7}{|c|}{ Gender } \\
\hline Male & 243 & 51.0 & $6.81(0.33)$ & 0.00 & - & 0.330 \\
\hline Female & 214 & 49.0 & $6.25(0.34)$ & $-0.52(0.53)$ & 0.330 & \\
\hline \multicolumn{7}{|l|}{ Race/ethnicity } \\
\hline White & 218 & 42.7 & $5.27(0.31)$ & 0.00 & - & $<0.001$ \\
\hline African-American & 114 & 22.5 & $7.90(0.46)$ & $2.63(0.43)$ & $<0.001$ & \\
\hline Hispanic & 47 & 10.5 & $7.18(0.59)$ & $1.90(0.55)$ & 0.001 & \\
\hline Asian/Other & 78 & 24.3 & $7.19(0.62)$ & $1.95(0.79)$ & 0.013 & \\
\hline \multicolumn{7}{|l|}{ Age } \\
\hline 11 years & 243 & 54.8 & $6.15(0.32)$ & 0.00 & - & 0.205 \\
\hline 12 years & 201 & 42.8 & $7.02(0.36)$ & $0.85(0.50)$ & 0.089 & \\
\hline 13 years & 13 & 2.4 & $7.02(1.31)$ & $0.66(1.29)$ & 0.607 & \\
\hline \multicolumn{7}{|c|}{ Pathway 1: Stressful life events } \\
\hline \multicolumn{7}{|c|}{ Number of life events in the past 6 months } \\
\hline $0-3$ & 179 & 44.9 & $4.66(0.34)$ & 0.00 & - & $<0.001$ \\
\hline $4-5$ & 125 & 26.5 & $6.79(0.45)$ & $2.12(0.53)$ & $<0.001$ & \\
\hline$\geqslant 6$ & 153 & 28.6 & $9.33(0.42)$ & $4.67(0.46)$ & $<0.001$ & \\
\hline \multicolumn{7}{|c|}{ Pathway 2: Family environment } \\
\hline \multicolumn{7}{|l|}{ Marital status of parents } \\
\hline Married & 307 & 70.8 & $6.14(0.29)$ & 0.00 & - & 0.021 \\
\hline Divorced/Separated & 150 & 29.2 & $7.49(0.40)$ & $1.34(0.58)$ & 0.021 & \\
\hline \multicolumn{7}{|c|}{ Number of adults in household } \\
\hline 1 & 119 & 24.0 & $7.82(0.46)$ & 0.00 & - & 0.006 \\
\hline$\geqslant 2$ & 336 & 76.1 & $6.12(0.27)$ & $-1.67(0.60)$ & 0.006 & \\
\hline \multicolumn{7}{|l|}{ Parental support $^{\mathrm{d}}$} \\
\hline High & 167 & 39.9 & $4.80(0.32)$ & 0.00 & - & $<0.001$ \\
\hline Medium & 153 & 34.1 & $6.81(0.43)$ & $2.00(0.49)$ & $<0.001$ & \\
\hline Low & 131 & 26.0 & $8.78(0.44)$ & $3.98(0.57)$ & $<0.001$ & \\
\hline \multicolumn{7}{|c|}{ Pathway 3: Neighborhood characteristics ${ }^{\mathrm{e}}$} \\
\hline \multicolumn{7}{|c|}{ Neighborhood median household income } \\
\hline High & 262 & 57.6 & $5.91(0.29)$ & 0.00 & - & 0.008 \\
\hline Low & 195 & 42.4 & $7.36(0.37)$ & $1.50(0.57)$ & 0.008 & \\
\hline \multicolumn{7}{|c|}{ Neighborhood aggravated assault rate } \\
\hline Low & 299 & 63.4 & $5.74(0.27)$ & 0.00 & - & $<0.001$ \\
\hline High & 158 & 36.6 & $7.89(0.41)$ & $2.20(0.47)$ & $<0.001$ & \\
\hline
\end{tabular}

${ }^{a}$ Mean C-DISC depressive symptom count within each category of the covariates and standard error of the mean.

b Regression coefficient associated with each category of the covariates in relation to C-DISC depressive symptom count and standard error of the regression coefficient.

c Two-tailed $p$-value from $t$-tests or analysis of variance.

d Parental support scale was categorized into thirds to create high, medium, and low levels.

e Neighborhood median household income and aggravated assault rate (per 1000 population) were dichotomized at the median to create high and low categories.

median income and aggravated assault rate, with an $12 \%$ change in the regression coefficient for low family income (Pathway 3: $b=2.06$ for low vs. high family income; $p=0.002$ ). Finally, in a model adjusting for all three hypothesized mediating pathways, as well as demographic characteristics, the effect of low family income on depressive symptoms was reduced by $67 \%$ (Total: $b=0.76$ for low vs. high family income; $p=0.371$ ). In this model, a greater number of stressors $(b=4.10$ for $\geqslant 6$ vs. $0-3$ stressors; $p<0.001)$ and having divorced or separated parents ( $b=1.11$ vs. married parents; $p=0.012$ ) were associated with an increased level of depressive symptoms, while higher levels of parental support were associated with a lower level of depressive symptoms $(b=-0.38$ for a one unit increase on the parental support subscale score; $p<0.001)$. Based on the log likelihood values for models 


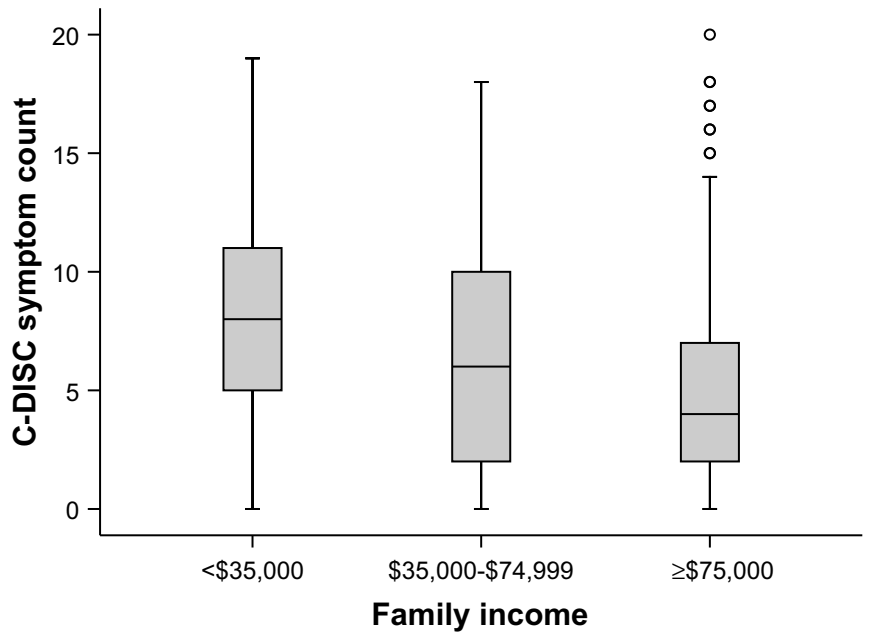

Fig. 2. Distribution of depressive symptoms by family income category.

entering each pathway in turn to the base model, the addition of Pathway 1 contributed significantly to model fit $(p<0.001)$, as did the addition of Pathway $2(p<0.001)$, and the addition of the three pathways simultaneously $(p<0.001)$, while the addition of Pathway 3 alone did not significantly improve model fit $(p=0.334)$.

\subsection{Sensitivity analyses}

We found a moderate but statistically significant correlation $(r=0.38 ; p<0.001)$ between the MASC total score and the C-DISC depressive symptoms count in our sample. Including the MASC total score as a measure of anxiety symptoms in the base model adjusted for family income, gender, race/ethnicity, and age, the effect of low family income on depressive symptoms was reduced by $26 \%$ when controlling for stressful life events, $40 \%$ when controlling for family environment, $13 \%$ when controlling for neighborhood characteristics, and $65 \%$ when controlling for all three pathways simultaneously. Anxiety symptoms were positively and significantly associated with depressive symptoms in all multivariable models.

Finally, when using a measure of family involvement rather than the parental support subscale, we found that the effect of low family income on depressive symptoms was attenuated by $35 \%$ when controlling for family environment, and higher levels of family involvement were significantly associated with lower levels of depressive symptoms. Complete results of the sensitivity analyses are available from the authors upon request.

\section{Discussion}

The mean depressive symptom count in our sample was comparable to reports from other samples of similarly aged children (Campbell and Ewing, 1990; Kim et al., 2003). Our findings are consistent with a number of studies suggesting that low family income is associated with higher levels of depressive symptoms in children and adolescents
(Call and Nonnemaker, 1999; Goodman et al., 2003; Goodman, 1999; Kubik et al., 2003; McLeod and Shanahan, 1993; Roberts et al., 1997; Schraedley et al., 1999; Wade, 2001). We evaluated three potential pathways that may explain this relation.

First, much evidence exists for the relation between stressful life events and depressive symptoms among adolescents (Franko et al., 2004; Nolen-Hoeksema et al., 1992; Patton et al., 2003; Schraedley et al., 1999; Silberg et al., 1999), and it has been suggested that even generally positive life events (e.g., birth of a new sibling) may serve as stressors, especially in middle-school-aged children who are undergoing rapid physical and emotional changes (Luthar, 1991). Consistent with this work, we found that experiencing a greater number of life events in the past 6 months was strongly associated with higher numbers of depressive symptoms among children. Although it has been hypothesized that life events may mediate the relation between low household SES and depressive symptoms in children (Costello et al., 2003; McLoyd, 1998), few studies have explicitly tested this hypothesis. One test of this hypothesis in the Great Smoky Mountains Study failed to find a mediating role of life stressors (e.g., parent separation or divorce), in the relation between resolution of poverty and total psychiatric symptoms among children aged 9-13 years at baseline (Costello et al., 2003). By contrast, we showed that adjusting for life events did attenuate the relation between low family income and depression, although the effect remained statistically significant, suggesting that greater exposure to adverse life events among children in low income families partially mediates the relation between family income and children's depressive symptoms. The differing urban/rural contexts of these studies, the differing outcomes of interest (total psychiatric symptoms as reported by children and their parents or caregivers in the Great Smoky Mountains Study versus child-reported depressive symptoms in our study), and the differing measures of SES used in these studies (resolution of poverty versus level of family income) may have contributed to the difference in results.

Second, one life stressor that may have a particular impact on the development of mental health symptoms in children and adolescents is the experience of parental divorce or separation (Aseltine, 1996; Gilman et al., 2003; Takeuchi et al., 1991; Wade, 2001), as well as other characteristics of the family environment. In our study, children whose parents were divorced or separated had a higher level of depressive symptoms, even after adjusting for level of parental support and number of adults present in the household. Financial hardship is likely both an antecedent and a consequence of family conflict and parental divorce (Aseltine, 1996; Gilman et al., 2003; McLoyd, 1990) supporting the hypothesis of the family environment as an overlapping risk factor with family income. Our study suggests that, consistent with some prior work (Gilman et al., 2003), the family environment may influence depressive symptoms over and above its association with family 
Table 2

Distribution of covariates of interest by family income and bivariate relations between covariates and family income

\begin{tabular}{|c|c|c|c|c|c|c|c|c|}
\hline & \multirow{2}{*}{$\begin{array}{l}\text { High income }{ }^{\mathrm{a}},{ }^{\mathrm{b}} N=146 \\
N(\%)\end{array}$} & \multicolumn{3}{|c|}{ Medium income ${ }^{\mathrm{b}} N=158$} & \multicolumn{3}{|c|}{ Low income ${ }^{\mathrm{b}} N=143$} & \multirow[t]{2}{*}{ Wald p-value } \\
\hline & & $N(\%)$ & $b(\mathrm{SE})^{\mathrm{c}}$ & $p$-value & $N(\%)$ & $b(\mathrm{SE})^{\mathrm{c}}$ & $p$-value & \\
\hline \multicolumn{9}{|l|}{ Demographic characteristics of students } \\
\hline \multicolumn{9}{|l|}{ Gender } \\
\hline Male & $86(55.9)$ & $80(46.2)$ & 0.00 & - & $72(50.5)$ & 0.00 & - & 0.334 \\
\hline Female & $60(44.1)$ & $78(53.8)$ & $0.39(0.23)$ & 0.089 & $71(49.5)$ & $0.22(0.23)$ & 0.350 & \\
\hline \multicolumn{9}{|l|}{ Race/ethnicity } \\
\hline White & $108(68.4)$ & $79(45.5)$ & 0.00 & - & $27(12.8)$ & 0.00 & - & $<0.001$ \\
\hline African-American & $4(2.3)$ & $37(22.4)$ & $2.71(0.59)$ & $<0.001$ & $70(43.0)$ & $4.63(0.61)$ & $<0.001$ & \\
\hline Hispanic & $17(12.0)$ & $10(5.6)$ & $-0.34(0.44)$ & 0.438 & 20 (14.9) & $1.90(0.41)$ & $<0.001$ & \\
\hline \multicolumn{9}{|l|}{ Age } \\
\hline 11 years & $80(56.6)$ & $81(53.3)$ & 0.00 & - & $74(52.7)$ & 0.00 & - & 0.628 \\
\hline 12 years & $64(42.4)$ & $74(44.1)$ & $0.10(0.23)$ & 0.675 & $61(43.5)$ & $0.10(0.24)$ & 0.682 & \\
\hline 13 years & $2(1.0)$ & $3(2.6)$ & $1.02(0.97)$ & 0.297 & $8(3.8)$ & $1.40(0.94)$ & 0.137 & \\
\hline \multicolumn{9}{|l|}{ Pathway 1: Stressful life events } \\
\hline \multicolumn{9}{|l|}{ Number of life events in the past 6 months } \\
\hline $0-3$ & $73(57.7)$ & $59(38.6)$ & 0.00 & - & $42(37.4)$ & 0.00 & - & $<0.001$ \\
\hline $4-5$ & $43(27.2)$ & $46(32.3)$ & $0.58(0.27)$ & 0.033 & 34 (20.3) & $0.14(0.30)$ & 0.631 & \\
\hline$\geqslant 6$ & $30(15.2)$ & $53(29.1)$ & $1.05(0.31)$ & 0.001 & $67(42.3)$ & $1.46(0.30)$ & $<0.001$ & \\
\hline \multicolumn{9}{|l|}{ Marital status of parents } \\
\hline Married & $113(78.4)$ & $113(74.0)$ & 0.00 & - & 74 (59.6) & 0.00 & - & 0.010 \\
\hline Divorced/Separated & 33 (21.6) & $45(26.0)$ & $0.24(0.27)$ & 0.373 & $69(40.4)$ & $0.90(0.26)$ & 0.001 & \\
\hline \multicolumn{9}{|l|}{ Number of adults in household } \\
\hline 1 & $14(9.2)$ & $30(16.8)$ & 0.00 & - & $72(47.0)$ & 0.00 & - & $<0.001$ \\
\hline$\geqslant 2$ & $132(90.8)$ & $127(83.2)$ & $-0.69(0.35)$ & 0.053 & $70(53.0)$ & $-2.17(0.33)$ & $<0.001$ & \\
\hline \multicolumn{9}{|l|}{ Parental support ${ }^{\mathrm{e}}$} \\
\hline High & $71(57.1)$ & $60(39.3)$ & 0.00 & - & $35(25.0)$ & 0.00 & - & $<0.001$ \\
\hline Medium & 46 (28.6) & $47(34.4)$ & $0.56(0.27)$ & 0.037 & $56(39.4)$ & $1.15(0.29)$ & $<0.001$ & \\
\hline Low & $27(14.3)$ & $49(26.3)$ & $0.98(0.32)$ & 0.002 & $50(35.6)$ & $1.74(0.33)$ & $<0.001$ & \\
\hline \multicolumn{9}{|l|}{ Pathway 3: Neighborhood characteristics } \\
\hline Mean median household income (SE) & $\$ 55,411(\$ 868)$ & $\$ 49,218(\$ 869)$ & $-0.72(0.13)$ & $<0.001$ & $\$ 45,174$ (\$944) & $-1.23(0.15)$ & $<0.001$ & $<0.001$ \\
\hline Mean aggravated assault rate $(\mathrm{SE})^{\mathrm{f}}$ & $2.14(0.19)$ & $4.12(0.67)$ & $0.33(0.06)$ & $<0.001$ & $5.28(0.54)$ & $0.37(0.06)$ & $<0.001$ & $<0.001$ \\
\hline
\end{tabular}

${ }^{\mathrm{a}}$ High family income serves as the referent group for bivariate polytomous logistic regression models with covariates as predictors of family income level.

b High family income corresponds to $\geqslant \$ 75,000$; medium family income to $\$ 35,000-\$ 74,999$; and low family income to $<\$ 35,000$.

c Regression coefficient associated with each category of the covariates in relation to medium and low (versus high) family income and standard error of the regression coefficient

d Two-tailed $p$-value from $\chi^{2}$ tests.

e Parental support scale was categorized into thirds to create high, medium, and low levels.

${ }^{\mathrm{f}}$ Mean aggravated assault rate is expressed per 1000 population. 
Table 3

Multivariable models of the relation between family income and depressive symptom

\begin{tabular}{|c|c|c|c|c|c|c|c|c|c|c|}
\hline & \multicolumn{2}{|l|}{ Base model } & \multicolumn{2}{|l|}{ Pathway 1} & \multicolumn{2}{|l|}{ Pathway 2} & \multicolumn{2}{|l|}{ Pathway 3} & \multicolumn{2}{|l|}{ Total } \\
\hline & $b(\mathrm{SE})^{\mathrm{a}}$ & $p$-value & $b(\mathrm{SE})^{\mathrm{a}}$ & $p$-value & $b(\mathrm{SE})^{\mathrm{a}}$ & $p$-value & $b(\mathrm{SE})^{\mathrm{a}}$ & $p$-value & $b(\mathrm{SE})^{\mathrm{a}}$ & $p$-value \\
\hline \multicolumn{11}{|l|}{ Family income } \\
\hline High $(\geqslant \$ 75,000)$ & 0.00 & - & 0.00 & - & 0.00 & - & 0.00 & - & 0.00 & - \\
\hline Medium $(\$ 35,000-\$ 74,999)$ & $1.04(0.52)$ & 0.046 & $0.46(0.54)$ & 0.395 & $0.64(0.49)$ & 0.189 & $0.89(0.47)$ & 0.060 & $0.13(0.48)$ & 0.785 \\
\hline Low $(<\$ 35,000)$ & $2.33(0.70)$ & 0.001 & $1.68(0.74)$ & 0.023 & $1.27(0.88)$ & 0.151 & $2.06(0.67)$ & 0.002 & $0.76(0.85)$ & 0.371 \\
\hline \multicolumn{11}{|l|}{ Demographic characteristics of students } \\
\hline \multicolumn{11}{|l|}{ Gender } \\
\hline Male & 0.00 & - & 0.00 & - & 0.00 & - & 0.00 & - & 0.00 & - \\
\hline Female & $-0.54(0.57)$ & 0.348 & $-0.29(0.50)$ & 0.571 & $-0.37(0.53)$ & 0.476 & $-0.39(0.57)$ & 0.491 & $-0.10(0.46)$ & 0.820 \\
\hline \multicolumn{11}{|l|}{ Race/ethnicity } \\
\hline White & 0.00 & - & 0.00 & - & 0.00 & - & 0.00 & - & 0.00 & - \\
\hline African-American & $1.27(0.62)$ & 0.041 & $0.52(0.63)$ & 0.410 & $1.09(0.63)$ & 0.084 & $1.22(0.65)$ & 0.061 & $0.59(0.64)$ & 0.360 \\
\hline Hispanic & $1.34(0.51)$ & 0.009 & $1.07(0.55)$ & 0.054 & $0.78(0.48)$ & 0.107 & $1.21(0.54)$ & 0.024 & $0.66(0.58)$ & 0.255 \\
\hline Asian/Other & $1.30(0.80)$ & 0.104 & $1.54(0.75)$ & 0.040 & $1.12(0.80)$ & 0.160 & $1.37(0.79)$ & 0.082 & $1.52(0.77)$ & 0.048 \\
\hline \multicolumn{11}{|l|}{ Age } \\
\hline 11 years & 0.00 & - & 0.00 & - & 0.00 & - & 0.00 & - & 0.00 & - \\
\hline 12 years & $0.81(0.50)$ & 0.104 & $0.34(0.45)$ & 0.451 & $0.46(0.49)$ & 0.341 & $0.68(0.49)$ & 0.163 & $0.05(0.41)$ & 0.912 \\
\hline 13 years & $-0.81(1.00)$ & 0.419 & $-0.45(0.80)$ & 0.575 & $-1.43(0.87)$ & 0.103 & $-1.05(1.03)$ & 0.310 & $-1.05(0.84)$ & 0.213 \\
\hline \multicolumn{11}{|l|}{ Pathway 1: Stressful life events } \\
\hline \multicolumn{11}{|l|}{ Number of life events in the past 6 months } \\
\hline $0-3$ & & & 0.00 & - & & & & & 0.00 & - \\
\hline $4-5$ & & & $2.59(0.49)$ & $<0.001$ & & & & & $2.31(0.48)$ & $<0.001$ \\
\hline$\geqslant 6$ & & & $4.52(0.52)$ & $<0.001$ & & & & & $4.10(0.50)$ & $<0.001$ \\
\hline \multicolumn{11}{|l|}{ Pathway 2: Family environment } \\
\hline \multicolumn{11}{|l|}{ Marital status of parents } \\
\hline Married & & & & & 0.00 & - & & & 0.00 & - \\
\hline Divorced/Separated & & & & & $1.14(0.56)$ & 0.041 & & & $1.11(0.44)$ & 0.012 \\
\hline \multicolumn{11}{|l|}{ Number of adults in household } \\
\hline 1 & & & & & 0.00 & - & & & 0.00 & - \\
\hline$\geqslant 2$ & & & & & $-0.54(0.71)$ & 0.449 & & & $-0.39(0.59)$ & 0.508 \\
\hline Parental support ${ }^{\mathrm{b}}$ & & & & & $-0.48(0.07)$ & $<0.001$ & & & $-0.38(0.07)$ & $<0.001$ \\
\hline \multicolumn{11}{|l|}{ Pathway 3: Neighborhood characteristics } \\
\hline Median household income (per $\$ 10,000$ ) & & & & & & & $-0.32(0.31)$ & 0.305 & $0.04(0.32)$ & 0.893 \\
\hline Aggravated assault rate (per 1,000 population) & & & & & & & $0.06(0.05)$ & 0.239 & $0.08(0.05)$ & 0.082 \\
\hline
\end{tabular}


income. The effect of the family environment, particularly with regard to parental divorce or separation, on depression may stem from exposure to prolonged conflict between custodial and non-custodial parents (Amato and Keith, 1991; Amato, 2000), less positive parenting and parental attention in divorced or separated families (Black and Pedro-Carroll, 1993; McLeod and Shanahan, 1993), or conflict with parents or new step-parents after the divorce (Aseltine, 1996; Leon, 2003). Additionally, it is possible that the observed relation reflects confounding by parental psychopathology, since parental divorce or separation is associated with a greater likelihood of depression among parents, and parental depression is related to depression in offspring (Spence et al., 2002; Wood et al., 2004). Higher levels of parental support were associated with lower levels of depressive symptoms in this analysis, independent of the marital status of the parents, which is consistent with other studies that have found a strong protective role of support from both custodial and non-custodial parents in predicting children's internalizing and externalizing behaviors (King and Sobolewski, 2006; Leon, 2003).

Third, in this sample, neighborhood characteristics were not associated with children's depressive symptoms once family income and other demographic characteristics had been taken into account, although they were significantly associated with depressive symptoms in bivariate models. These findings support the hypothesis that neighborhood characteristics like median household income and level of violent crime may serve as proxy risk factors, which are only associated with depressive symptoms through their relation with family income. However, other studies have observed a significant effect of neighborhood deprivation on internalizing symptoms or behavior problems in younger age groups, even after adjusting for individual-level SES and other demographic characteristics (Caspi et al., 2000; Kalff et al., 2001; Xue et al., 2005), though few studies have evaluated the influence of neighborhood residence on the risk of depression among older children using objective measures of neighborhood characteristics. The Moving to Opportunity Study demonstrated that children whose families moved from high- to low-poverty neighborhoods experienced significant declines in anxious and depressive symptoms, compared to children in families who remained in impoverished neighborhoods, but only among boys (Leventhal and Brooks-Gunn, 2003). Since middleschool-aged children may be starting to spend more time in their neighborhood than in their homes (Caspi et al., 2000), further research is needed to determine whether other characteristics of the neighborhood environment not assessed in this study, including poor physical conditions and lack of availability of social and other resources, influence child mental health status apart from their relation with family income and to determine whether any effects are consistently stronger for boys, who may have greater exposure to neighborhood conditions and who may be more vulnerable to stressors (Bolger et al., 1995; Leventhal and Brooks-Gunn, 2003).
The three hypothesized pathways between family income and depressive symptoms tested in this analysis are substantially related to each other (Amato, 2000; Aseltine, 1996; South et al., 1998). Simultaneously controlling for all three pathways attenuated the effect of low family income on depression by about two-thirds but had only a minor impact on the effects of life stressors, parental marital status, and parental support, demonstrating the importance of these factors to children's depressive symptoms.

This study had several limitations. Because of the low prevalence of major depressive disorder in this young sample, depressive symptom count was used as the outcome of interest. Inferences from this analysis with regard to predictors of increased levels of depressive symptoms and the pathways through which family income influences depressive symptoms may not translate directly when looking specifically at depressive disorders. However, the extant literature suggests that symptom count is relevant for identifying youths at high risk of developing depressive disorders (Fergusson et al., 2005) and other adverse outcomes (Kim et al., 2003; Vander Stoep et al., 2002), and that the continuous nature of depressive symptoms is important to consider (Fergusson et al., 2005; Kim et al., 2003); as such, these findings may provide useful insight into the factors influencing depression in childhood and adolescence. The cross-sectional nature of this analysis limits the causal inferences that can be made, since the timing of onset of depressive symptoms cannot be assessed in relation to family income and other factors examined in this study. However, the drift phenomenon, whereby persons experience loss of economic status as a result of their mental health status (Dohrenwend et al., 1992) is probably not operative in child populations. No information on long-term income history was available for study subjects, so we were unable to differentiate between persistent and short-term experiences of poverty and their respective associations with depressive symptoms. We were also unable to assess the role of parenting behaviors, parental conflict, and parental psychopathology as other indicators of the family environment that may explain the relation between low family income and depressive symptoms. Perceptions of parental support may have been influenced by depressive symptoms; however, use of a more objective measure of family involvement resulted in similar findings for the relation between support and depressive symptoms and the attenuation of the effect of low family income on depressive symptoms when controlling for indicators of the family environment. The neighborhood units used in our analysis were fairly large, and we cannot exclude the possibility that neighborhood characteristics at a smaller geographic neighborhood level may play a stronger role in determining childhood depression. Neighborhood income and aggravated assault rate were used to test the hypothesized role of the neighborhood environment as a proxy risk factor in the relation between family income and depressive symptoms. Although other salient neighborhood characteristics (e.g., unemployment, poor walkability, high residential 
instability, poor built environment) are highly correlated with neighborhood income and violent crime, it is possible that one or more of these other measures may have provided a better test of this hypothesized pathway.

All information about children and their households was collected via self-report in this study. The sample was recruited through a public-school-based screening program and as such excludes children who attend private schools, are home-schooled, or are out of school due to running away, homelessness, juvenile delinquency, or chronic illness. Although there were some demographic differences between eligible students who did and did not participate in the baseline interview of the longitudinal study, the sample was representative of the Seattle public middle school population after application of weights so these differences are not likely to have influenced our findings. Finally, this study was conducted among a diverse population of sixth grade students in a moderately large urban area in the United States, and results may not be generalizable to other age groups, population samples, and geographic areas.

Notwithstanding these limitations, our study shows that the strong relation between family income and depressive symptoms in middle-school-aged children can be explained in part by the greater likelihood that children in low income families are exposed to stressful life events, experience family environments characterized by parental divorce or separation and lower levels of parental support, and reside in lower-income and higher-crime neighborhoods. Further exploration of the pathways between family SES and depression will provide insight into potential interventions that may reduce the occurrence and persistence of depressive symptoms among the large number of children living in poverty in the United States and worldwide.

\section{Conflict of interest}

All authors declare that they have no conflicts of interest.

\section{Role of funding source}

This work was supported by a grant from the National Institute of Mental Health and the National Institute of Drug Abuse (R01 MH63711, Ann Vander Stoep, PI). These agencies had no further role in study design; in the collection, analysis and interpretation of data; in the writing of the manuscript; and in the decision to submit the manuscript for publication.

\section{Contributors}

M. Tracy analyzed the data and wrote the manuscript. F. Zimmerman and S. Galea contributed to data analysis and manuscript preparation. E. McCauley and A. Vander Stoep designed and supervised the study and contributed to manuscript preparation.

\section{Acknowledgements}

This work was supported by a grant from the National Institute of Mental Health and the National Institute of Drug Abuse (R01 MH63711, Ann Vander Stoep, PI).

\section{References}

Achenbach TM. Youth self report (YSR). Burlington, VT: University of Vermont; 2001.

Amato PR. The consequences of divorce for adults and children. Journal of Marriage and the Family 2000;62:1269-87.

Amato PR, Keith B. Parental divorce and the well-being of children: a meta-analysis. Psychological Bulletin 1991;110:26-46.

American Psychiatric Association, Task force on DSM-IV. Diagnostic and statistical manual of mental disorders: DSM-IV. 4th ed. Washington (DC): American Psychiatric Association; 1994.

Angold A, Costello EJ. Mood and feelings questionnaire (MFQ). Durham (NC): Duke University, Developmental Epidemiology Program; 1987.

Angold A, Weissman MW, John K, Merikangas KR, Prusoff BA, Wickramaratne $\mathrm{P}$, et al. Parent and child reports of depressive symptoms in children at low and high risk of depression. Journal of Child Psychology and Psychiatry, and Allied Disciplines 1987;28:901-15.

Aseltine Jr RH. Pathways linking parental divorce with adolescent depression. Journal of Health and Social Behavior 1996;37:133-48.

Baldwin JS, Dadds MR. Reliability and validity of parent and child versions of the Multidimensional Anxiety Scale for Children in community samples. Journal of the American Academy of Child and Adolescent Psychiatry 2007;46:252-60.

Belsher G, Costello CG. Relapse after recovery from unipolar depression: a critical review. Psychological Bulletin 1988;104:84-96.

Birmaher B, Ryan ND, Williamson DE, Brent DA, Kaufman J, Dahl RE, et al. Childhood and adolescent depression: a review of the past 10 years. Part I. Journal of the American Academy of Child and Adolescent Psychiatry 1996;35:1427-39.

Black AE, Pedro-Carroll J. Role of parent-child relationships in mediating the effects of marital disruption. Journal of the American Academy of Child and Adolescent Psychiatry 1993;32:1019-27.

Bolger KE, Patterson CJ, Thompson WW, Kupersmidt JB. Psychosocial adjustment among children experiencing persistent and intermittent family economic hardship. Child Development 1995;66:1107-29.

Bradley RH, Corwyn RF. Socioeconomic status and child development. Annual Review of Psychology 2002;53:371-99.

Brady EU, Kendall PC. Comorbidity of anxiety and depression in children and adolescents. Psychological Bulletin 1992;111:244-55.

Brooks-Gunn J, Duncan GJ, Klebanov PK, Sealand N. Do neighborhoods influence child and adolescent development? American Journal of Sociology 1993;99:353-95.

Call KT, Nonnemaker J. Socioeconomic disparities in adolescent health: contributing factors. Annals of the New York Academy of Sciences 1999;896:352-5.

Campbell SB, Ewing LJ. Follow-up of hard-to-manage preschoolers: adjustment at age 9 and predictors of continuing symptoms. Journal of Child Psychology and Psychiatry, and Allied Disciplines 1990;31: 871-89.

Caspi A, Taylor A, Moffitt TE, Plomin R. Neighborhood deprivation affects children's mental health: environmental risks identified in a genetic design. Psychological Science 2000;11:338-42.

Cawthorpe D. An evaluation of a computer-based psychiatric assessment: evidence for expanded use. Cyberpsychology and Behavior 2001;4: 503-10.

Costello EJ, Edelbrock CS, Costello AJ. Validity of the NIMH Diagnostic Interview Schedule for Children: a comparison between psychiatric and pediatric referrals. Journal of Abnormal Child Psychology 1985;13:579-95. 
Costello EJ, Compton SN, Keeler G, Angold A. Relationships between poverty and psychopathology: a natural experiment. Journal of the American Medical Association 2003;290:2023-9.

Department of City Planning, City of Seattle. Census 2000 data for locally defined areas: Community Reporting Areas, 2000. Available fron: www.seattle.gov/DPD/demographics/data_local_cra.asp.

Department of Neighborhoods, City of Seattle. Community Reporting Areas for Seattle \& Census 2000 Tracts, 2000. Available from: www.seattle.gov/DPD/demographics/census_local_cra/maps_aLL_ cra_tract00.pdf.

Dierker LC, Albano AM, Clarke GN, Heimberg RG, Kendall PC, Merikangas KR, et al. Screening for anxiety and depression in early adolescence. Journal of the American Academy of Child and Adolescent Psychiatry 2001;40:929-36.

Dohrenwend BP, Levav I, Shrout PE, Schwartz S, Naveh G, Link BG, et al. Socioeconomic status and psychiatric disorders: the causationselection issue. Science 1992;255:946-52.

Dooley D, Catalano R, Wilson G. Depression and unemployment: panel findings from the Epidemiologic Catchment Area study. American Journal of Community Psychology 1994;22:745-65.

Edelbrock C, Costello AJ, Dulcan MK, Kalas R, Conover NC. Age differences in the reliability of the psychiatric interview of the child. Child Development 1985;56:265-75.

Edelbrock C, Costello AJ, Dulcan MK, Conover MC, Kalas R. Parentchild agreement of child psychiatric symptoms assessed via structured interview. Journal of Child Psychology and Psychiatry, and Allied Disciplines 1986;27:181-90.

Federal Bureau of Investigations (FBI). Uniform crime reporting handbook, 2004. Available from: www.fbi.gov/ucr/handbook/ucrhandbook04.pdf.

Fergusson DM, Horwood LJ, Ridder EM, Beautrais AL. Subthreshold depression in adolescence and mental health outcomes in adulthood. Archives of General Psychiatry 2005;62:66-72.

Franko DL, Striegel-Moore RH, Brown KM, Barton BA, McMahon RP, Schreiber GB, et al. Expanding our understanding of the relationship between negative life events and depressive symptoms in black and white adolescent girls. Psychological Medicine 2004;34: 1319-30.

Gilman SE, Kawachi I, Fitzmaurice GM, Buka SL. Family disruption in childhood and risk of adult depression. American Journal of Psychiatry 2003;160:939-46.

Goodman E. The role of socioeconomic status gradients in explaining differences in US adolescents' health. American Journal of Public Health 1999;89:1522-8.

Goodman E, Huang B, Wade TJ, Kahn RS. A multilevel analysis of the relation of socioeconomic status to adolescent depressive symptoms: does school context matter? Journal of Pediatrics 2003;143: 451-6.

Graham CA, Easterbrooks MA. School-aged children's vulnerability to depressive symptomatology: the role of attachment security, maternal depressive symptomatology, and economic risk. Development and Psychopathology 2000;12:201-13.

Harter S. Manual for the social support scale for children and adolescents. Denver (CO): University of Denver; 1985.

Ivarsson T. Normative data for the Multidimensional Anxiety Scale for Children (MASC) in Swedish adolescents. Nordic Journal of Psychiatry 2006;60:107-13.

Johnson JH, Sarason IG. Recent developments in research on life stress. In: Hamilton V, Warburton DM, editors. Human stress and cognition: an information processing approach. London: Wiley Press; 1979.

Johnson JH, McCutcheon S. Assessing life stress in older children and adolescents: preliminary findings with the LEC. In: Sarason IG, Spielberger CD, editors. Stress and anxiety. Washington (DC): Hemisphere; 1980.

Kalff AC, Kroes M, Vles JS, Hendriksen JG, Feron FJ, Steyaert J, et al. Neighbourhood level and individual level SES effects on child problem behaviour: a multilevel analysis. Journal of Epidemiology and Community Health 2001;55:246-50.
Kelder SH, Murray NG, Orpinas P, Prokhorov A, McReynolds L, Zhang $\mathrm{Q}$, et al. Depression and substance use in minority middle-school students. American Journal of Public Health 2001;91:761-6.

Kessler RC, Walters EE. Epidemiology of DSM-III-R major depression and minor depression among adolescents and young adults in the National Comorbidity Survey. Depression and Anxiety 1998;7:3-14.

Kim IJ, Ge X, Brody GH, Conger RD, Gibbons FX, Simons RL. Parenting behaviors and the occurrence and co-occurrence of depressive symptoms and conduct problems among African-American children. Journal of Family Psychology 2003;17:571-83.

King V, Sobolewski JM. Nonresident fathers' contributions to adolescent well-being. Journal of Marriage and the Family 2006;68:537-57.

Kraemer HC, Stice E, Kazdin A, Offord D, Kupfer D. How do risk factors work together? Mediators, moderators, and independent, overlapping, and proxy risk factors. American Journal of Psychiatry 2001;158:848-56.

Kubik MY, Lytle LA, Birnbaum AS, Murray DM, Perry CL. Prevalence and correlates of depressive symptoms in young adolescents. American Journal of Health Behavior 2003;27:546-53.

Leech SL, Larkby CA, Day R, Day NL. Predictors and correlates of high levels of depression and anxiety symptoms among children at age 10 . Journal of the American Academy of Child and Adolescent Psychiatry 2006;45:223-30.

Leon K. Risk and protective factors in young children's adjustment to parental divorce: a review of the research. Family Relations 2003;52:258-70.

Leventhal T, Brooks-Gunn J. The neighborhoods they live in: the effects of neighborhood residence on child and adolescent outcomes. Psychological Bulletin 2000;126:309-37.

Leventhal T, Brooks-Gunn J. Moving to opportunity: an experimental study of neighborhood effects on mental health. American Journal of Public Health 2003;93:1576-82.

Lewinsohn PM, Rohde P, Seeley JR. Major depressive disorder in older adolescents: prevalence, risk factors, and clinical implications. Clinical Psychology Review 1998;18:765-94.

Lorant V, Deliege D, Eaton W, Robert A, Philippot P, Ansseau M. Socioeconomic inequalities in depression: a meta-analysis. American Journal of Epidemiology 2003;157:98-112.

Luthar SS. Vulnerability and resilience: a study of high-risk adolescents. Child Development 1991;62:600-16.

March JS, Parker JDA, Sullivan K, Stallings P, Conners CK. The Multidimensional Anxiety Scale for Children (MASC): Factor structure, reliability, and validity. Journal of the American Academy of Child and Adolescent Psychiatry 1997;36:554-65.

March JS, Sullivan K, Parker J. Test-retest reliability of the Multidimensional Anxiety Scale for Children. Journal of Anxiety Disorders 1999;13:349-58.

McLeod JD, Shanahan MJ. Poverty, parenting, and children's mental health. American Sociological Review 1993;58:351-66.

McLoyd VC. The impact of economic hardship on black families and children: psychological distress, parenting, and socioemotional development. Child Development 1990;61:311-46.

McLoyd VC. Socioeconomic disadvantage and child development. The American Psychologist 1998;53:185-204.

McQuillan K. Falling behind: the income of lone-mother families, 19701985. Canadian Review of Sociology and Anthropology 1992;29:511-23.

National Institute of Mental Health (NIMH). Computerized diagnostic interview schedule for children, IV (NIMH C-DISC IV). New York: Columbia University; 1997.

National Institute of Mental Health (NIMH). Section IV, Module C. In: Generic DISC 4 scoring manual. New York (NY): Columbia University; 2003.

Nolen-Hoeksema S, Girgus JS, Seligman ME. Predictors and consequences of childhood depressive symptoms: a 5-year longitudinal study. Journal of Abnormal Psychology 1992;101:405-22.

Ozer EJ, Weinstein RS. Urban adolescents' exposure to community violence: the role of support, school safety, and social constraints in a 
school-based sample of boys and girls. Journal of Clinical Child and Adolescent Psychology ;33:463-76.

Patton GC, Coffey C, Posterino M, Carlin JB, Bowes G. Life events and early onset depression: cause or consequence? Psychological Medicine 2003;33:1203-10.

Pine DS, Cohen P, Gurley D, Brook J, Ma Y. The risk for earlyadulthood anxiety and depressive disorders in adolescents with anxiety and depressive disorders. Archives of General Psychiatry 1998;55:56-64.

Roberts RE, Roberts CR, Chen YR. Ethnocultural differences in prevalence of adolescent depression. American Journal of Community Psychology 1997;25:95-110.

Ryan ND. Treatment of depression in children and adolescents. Lancet 2005;366:933-40.

Rynn MA, Barber JP, Khalid-Khan S, Siqueland L, Dembiski M, McCarthy KS, et al. The psychometric properties of the MASC in a pediatric psychiatric sample. Journal of Anxiety Disorders 2006;20:139-57.

Schraedley PK, Gotlib IH, Hayward C. Gender differences in correlates of depressive symptoms in adolescents. Journal of Adolescent Health 1999;25:98-108.

Schwab-Stone ME, Shaffer D, Dulcan MK, Jensen PS, Fisher P, Bird HR, et al. Criterion validity of the NIMH Diagnostic Interview Schedule for Children Version 2. 3 (DISC-2.3). Journal of the American Academy of Child and Adolescent Psychiatry 1996;35:878-88.

Seattle Police Department. Seattle Police 2000 Annual Report, 2000. $<$ www.seattle.gov/Police/Publications/AR/AR00.pdf $>$.

Seattle Public Schools. Data profile: district summary, December 2004. Available from: <www.seattleschools.org/area/siso/disprof/2004/ DP04all.pdf $>$.

Shaffer D, Fisher P, Lucas CP, Dulcan MK, Schwab-Stone ME. NIMH Diagnostic Interview Schedule for Children Version IV (NIMH DISCIV): description, differences from previous versions, and reliability of some common diagnoses. Journal of the American Academy of Child and Adolescent Psychiatry 2000;39:28-38.

Shaffer D, Scott M, Wilcox H, Maslow C, Hicks R, Lucas CP, et al. The Columbia Suicide Screen: Validity and reliability of a screen for youth suicide and depression. Journal of the American Academy of Child and Adolescent Psychiatry 2004;43:71-9.

Shah B, Barnwell B, Bieler G. SUDAAN user's manual, release 7.5. Research Triangle Park (NC): Sage; 1997.
Silberg J, Pickles A, Rutter M, Hewitt J, Simonoff E, Maes H, et al. The influence of genetic factors and life stress on depression among adolescent girls. Archives of General Psychiatry 1999;56:225-32.

Slesnick N, Prestopnik J. Dual and multiple diagnosis among substance using runaway youth. The American Journal of Drug and Alcohol Abuse 2005;31:179-201.

South SJ, Crowder KD, Trent K. Children's residential mobility and neighborhood environment following parental divorce and remarriage. Social Forces 1998;77:667-93.

Spence SH, Najman JM, Bor W, O'Callaghan MJ, Williams GM. Maternal anxiety and depression, poverty and marital relationship factors during early childhood as predictors of anxiety and depressive symptoms in adolescence. Journal of child psychology and psychiatry, and allied disciplines 2002;43:457-69.

Takeuchi DT, Williams DR, Adair RK. Economic stress in the family and children's emotional and behavioral problems. Journal of Marriage and the Family 1991;53:1031-41.

Twenge JM, Nolen-Hoeksema S. Age, gender, race, socioeconomic status, and birth cohort differences on the children's depression inventory: a meta-analysis. Journal of Abnormal Psychology 2002;111:578-88.

Vander Stoep A, Weiss NS, McKnight B, Beresford SAA, Cohen P. Which measure of adolescent psychiatric disorder - diagnosis, number of symptoms, or adaptive functioning - best predicts adverse young adult outcomes? Journal of Epidemiology and Community Health 2002;56:56-65.

Vander Stoep A, McCauley E, Thompson KA, Herting JR, Kuo ES, Stewart DG, et al. Universal emotional health screening during the middle school transition. Journal of Emotional and Behavioral Disorders 2005; 13:213-23.

Wade TJ. Delinquency and health among adolescents: multiple outcomes of a similar social and structural process. International Journal of Law and Psychiatry 2001;24:447-67.

Wood JJ, Repetti RL, Roesch SC. Divorce and children's adjustment problems at home and school: the role of depressive/withdrawn parenting. Child Psychiatry and Human Development 2004;35:121-42.

Xue Y, Leventhal T, Brooks-Gunn J, Earls FJ. Neighborhood residence and mental health problems of 5- to 11-year-olds. Archives of General Psychiatry 2005;62:554-63.

Zimmerman MA, Salem DA, Maton KI. Family structure and psychosocial correlates among urban African-American adolescent males. Child Development 1995;66:1598-613. 